\title{
A Survey on Healthy Food Decision Influences Through Technological Innovations
}

\author{
JERMAINE MARSHALL, PRISCILLA JIMENEZ-PAZMINO, RONALD METOYER, and NITESH \\ V. CHAWLA, Department of Computer Science \& Engineering, Lucy Family Institute for Data \& Society, \\ University of Notre Dame, USA
}

\begin{abstract}
It is well known that unhealthy food consumption plays a significant role in dietary and lifestyle-related diseases. Therefore, it is important for researchers to examine methods that may encourage the consumer to consider healthier dietary and lifestyle habits as diseases such as obesity, heart disease, and high blood pressure remain a worldwide issue. One promising approach to influencing healthy dietary and lifestyle habits is food recommendation models that recommend food to users based on various factors such as health effects, nutrition, preferences, and daily habits. Unfortunately, much of this work has focused on individual factors such as taste preferences and often neglects to understand other factors that influence our choices. Additionally, the evaluation of technological approaches often lacks user studies in the context of intended use. In this systematic review of food choice technology, we focus on the factors that may influence food choices and how technology can play a role in supporting those choices. We also describe existing work, approaches, trends, and issues in current food choice technology and give advice for future work areas in this space.
\end{abstract}

CCS Concepts: • General and reference $\rightarrow$ Surveys and overviews; $\bullet$ Social and professional topics $\rightarrow$ User characteristics;

Additional Key Words and Phrases: Food, health, environment, food properties, personal factors, technology, food decisions, food recommendation systems, health applications

ACM Reference format:

Jermaine Marshall, Priscilla Jimenez-Pazmino, Ronald Metoyer, and Nitesh V. Chawla. 2022. A Survey on Healthy Food Decision Influences Through Technological Innovations. ACM Trans. Comput. Healthcare 3, 2, Article 25 (February 2022 ), 27 pages.

https://doi.org/10.1145/3494580

\section{INTRODUCTION}

Given the variety of food options available today, sorting out the healthy foods from the unhealthy and making smart dietary and lifestyle decisions has become a challenge. From 1964 to 2015, food consumption increased

Jermaine Marshall was supported by the National Science Foundation (NSF) Graduate Research Fellowships Program. This research was also supported in part by the NSF Grant IIS-1447795.

Authors' addresses: J. Marshall, Department of Computer Science \& Engineering, Lucy Family Institute for Data \& Society, University of Notre Dame, 384E Nieuwland Science Hall, Notre Dame IN 46556, Notre Dame, IN, 46556, USA; email: jmarsha5@nd.edu; P. Jimenez-Pazmino, Department of Computer Science \& Engineering, Lucy Family Institute for Data \& Society, University of Notre Dame, 384E Nieuwland Science Hall, Notre Dame IN 46556, Notre Dame, IN, USA; email: pjimenez@nd.edu; R. Metoyer, Department of Computer Science \& Engineering, Lucy Family Institute for Data \& Society, University of Notre Dame, 384E Nieuwland Science Hall, Notre Dame IN 46556, Notre Dame, IN, USA; email: rmetoyer@nd.edu; N. V. Chawla, Department of Computer Science \& Engineering, Lucy Family Institute for Data \& Society, University of Notre Dame, 384E Nieuwland Science Hall, Notre Dame IN 46556, 384E Nieuwland Science Hall, Notre Dame, IN, USA; email: nchawla@nd.edu.

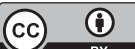 \\ This work is licensed under a Creative Commons Attribution International 4.0 License. \\ (C) 2022 Copyright held by the owner/author(s). \\ 2637-8051/2022/02-ART25 \$15.00 \\ https://doi.org/10.1145/3494580
}


from 2358 kilo-calories per capita per day to 2940 kilo-calories per capita per day with the majority of dietary fat being consumed in North America and Europe [78]. In 2016, over 2 billion people over the age of 18 years had been diagnosed as being overweight with 650 million of those being considered as obese. Through careful analysis of the health data from approximately 190 countries, researchers discovered that high blood pressure and heart disease affect approximately 1.1 billion people each year and kill 17.3 million people each year, respectively. Heart disease was also discovered to be the leading cause of death in these countries [4, 77, 79].

Due to the higher number of people with diet-related diseases, it becomes important to ensure that consumers are getting adequate nutrition. The Internet provides easy access to online recipes to make at home. However, according to a study by Trattner and Elsweiler [69], the majority of online recipes tend to be unhealthy - with many, for example, having excess amounts of sodium.

Particular diet-related health diseases, such as obesity, can also cause mental health issues. In a study by Devlin et al. [18], it was revealed that obesity can cause negative side effects such as problems getting married, which, in turn, can lead to depression. Scott et al. also performed a study showing that obesity, particularly severe obesity, had negative impacts on the mental health of individuals, with a stronger effect on women [56].

Food choice technologies may be able to support healthy food selection. Many Americans desire the benefits from consumption of a healthy diet but often struggle to find and consume foods that contain specific benefits for their particular health-related illness [26]. For example, it may be difficult for the average consumer to know the difference in nutritional value between organic and non-organic potato chips (if any) or the consumer may not like the taste of particular nutritional foods (e.g., broccoli) and, thus, are hesitant to consume them. There is now an opportunity to develop algorithms and machine learning techniques that aid consumers in understanding what healthy choices are available and in understanding what they should potentially be consuming.

\subsection{Existing Surveys}

DiFilippo et al. [19] conducted a survey on mobile technologies - particularly nutrition apps -and discussed their usefulness for increasing nutrition knowledge to improve dietary behavior, with a focus on weight loss. Tonkin et al. [66] conducted a literature review on the use of smart-phone applications for nutrition improvement in community settings. They discussed the methods for consumer testing in applications that were aimed at nutrition improvement in communities. Nour et al. [46] took a similar approach but instead focused on mobile technologies designed to improve vegetable intake in young adults. Our review does not focus specifically on apps or on a particular type of food or user population. Rather, we are interested in focusing on factors and context of use, with an eye on recommendation technology.

Several reviews have also focused on food recommendation technology. Tran et al. [67] developed a survey that covered the different types of recommendation systems, such as collaborative filtering, content-based recommendations, and hybrid recommendations, with respect to the healthy food recommendation system literature. The review focused on the details of how researchers performed healthy food recommendations using machine learning algorithms. They presented an in-depth discussion on the importance of user preferences (e.g., taste and ingredients) and constraints (e.g., nutritious needs, dietary restrictions, and cost of food) in the recommendation model. Additionally, the authors highlighted the challenges of the research. These challenges included "collecting user information, gathering more nutritious information for recipes, advancing recommendation algorithms, explaining why recommendations were made, and changing the current eating behaviors of the user to comply with healthy behaviors" [67]. Trattner et al. [68] also performed a literature review that gave their perspective on future directions for food recommendation systems.

A key difference between the survey by Tran et al. [67] and ours is that we focus on reviewing technological approaches that aid in healthy food choices along with the variables and contexts considered while Tran et al. focus only on the types of algorithms used for food recommendations. We differ from Trattner et al. [68] in a similar light as they mainly focus on frameworks developed for food recommendation systems as well as the algorithms utilized to build and evaluate them. 
To the best of our knowledge, this is the first survey that focuses on food choice technologies that aim to support healthy food choices without restricting the scope of the review to recommendation systems only. Prior work on food choice technologies has focused primarily on personal preferences, such as taste, lifestyle habits, and affinity for nutritious ingredients. Prior literature reviews on the topic of food choice have examined technologies such as mobile devices, e-health applications, and gaming as well as how each may influence healthy food choices [2, 8, 33, 82]. We also discovered a review by Roberto et al. [53] on obesity prevention that discussed barriers to prevention of obesity. The author mentions that of the current existing approaches, targeting user influences may be one of the most effective methods to halt excessive weight gain.

In this review, we focus on examining factors that are considered in the design of food choice technologies. We also examine how those factors align with what is already known about elements taken into consideration by consumers when making food choices and in what contexts these choices are made. In particular, we ask the following five research questions:

RQ1: Which factors/variables have been considered in healthy food choice technologies?

RQ2: Which contexts of use have been explored?

RQ3: What are the technological approaches for food recommendations?

RQ4: Which dataset types are the main sources of input for food choice technology?

RQ5: What are the most common evaluation metrics for food choice technology?

The goal of this work is to highlight the gaps in the research and complement existing work in the healthy food choice space by showing how different techniques, themes, and context can be used together to aid in providing food choice guidance to consumers in general.

We expand on prior work with the following contributions. First, we identify the most common food choice factors and context considered in current food choice technology solutions. As we will discuss, many social, cultural, and environmental factors of food choice are under-explored in today's research. Second, we discuss common practices for influencing healthy food consumption from a technological perspective, how to incorporate influential factors by utilizing modern machine-learning approaches, and how to effectively evaluate healthy food choice technologies. Finally, we consolidate and discuss important food-choice technology datasets as well as discuss possibilities for directions of future work. ${ }^{1}$

\section{SURVEY APPROACH}

In this section, we discuss the process used for our literature review, including the topic we are covering, the consulted databases indexed, the search terms used, the selection and filtering process, and the analysis process.

\subsection{Scope of the Survey}

In this survey, we focus on healthy food choice technologies to gain insight on what factors have been considered when recommending nutrition-related information to consumers.

We particularly focus on influencing and recommending healthy food choices through technology to identify how to support more personalized recommendations. We do not consider work that focuses on identifying food via image recognition (e.g., Rich et al. [52] and Chen et al. [11]) as it does not pertain to recommendations or influence.

Addtionally, this review emphasizes healthy food choice through technology. We excluded papers that focused solely on food recommendations (e.g., Doerr et al. [20]) that do not consider nutrition or health.

Identifying and categorizing factors that determine eating behavior is a complex task. The outcome has usually been discipline oriented. In an attempt to provide an interdisciplinary and dynamic framework, Stok et al. [62] proposed the DONE (Determinants Of Nutrition and Eating behavior) framework (Figure 2), which has considered input from 10 different disciplines. This framework not only provides us with a taxonomy and structure

\footnotetext{
${ }^{1}$ http://techfoodsurvey.site.
} 


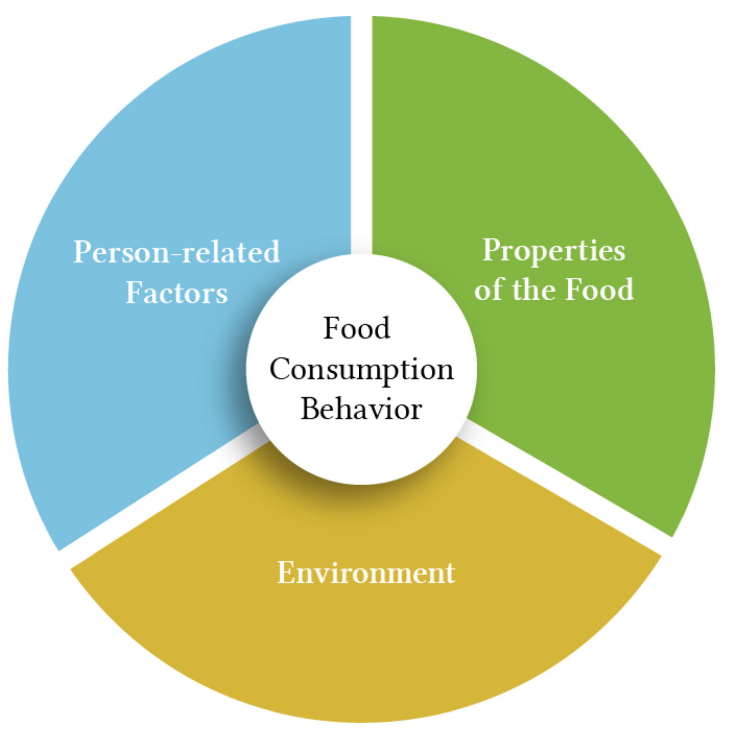

(a) Categories of factors suggested by consumer researchers $[40,61,63]$

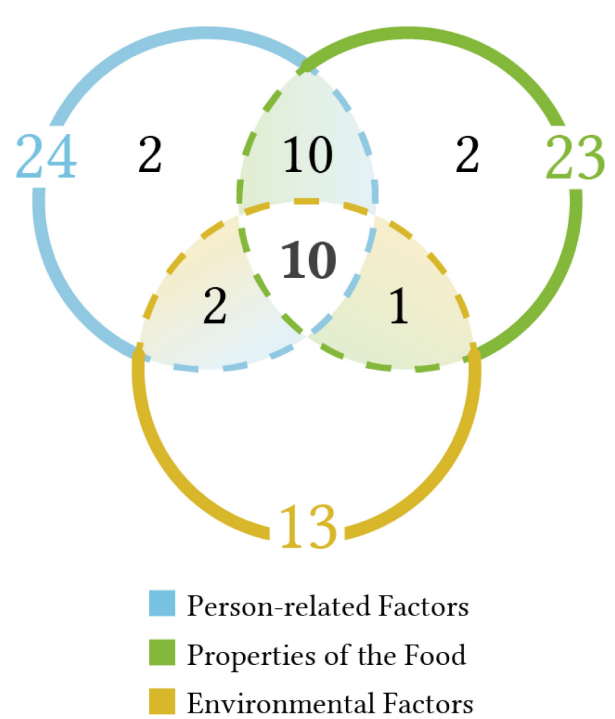

(b) Count of papers in the review by Categories

Fig. 1. Categories of factors that determine food consumption.

that will be used under the three identified themes but also provides guidance to discuss the potential priorities for research in food choice technology design.

We also decided to place the articles into categories based on the context of each article's target audience. This organization into context of use categories (i.e., personal, social, and grocery) was done for clarity and to explain how these contexts may affect food choices.

\subsection{Databases Indexed}

The articles considered for this review were gathered from the Association for Computing Machinery (ACM), the Institute of Electrical and Electronic Engineers (IEEE) digital libraries, and various medical journals, which included PLOS, Lancet, and the fournal of Medical Internet Research (FMIR). The ACM and IEEE represent leading computer science venues while PLOS, Lancet, and FMIR represent the leading medical journals. We note that this study takes place from October 2017 to May 2018; thus, we considered only those studies published up until May 2018. Papers were reviewed by the first two authors. The results we received from our searches were obtained by searching the metadata of the articles.

\subsection{Search Terms}

We used five main keywords that we deemed to be the most relevant to our topic of interest and followed certain criteria. First, we wanted to make sure that we included words relating to common outside factors in relation to food recommendations: culture, nutrients, and taste. Additionally, the metadata from our search had to contain both keywords food and health as we wanted results that specifically addressed the health of a consumer. For example, for the ACM database, the search was performed in the following format: "(+food thealth culture nutrients taste)".

We did not include the term recommendation in our search as that seemed to disqualify results that may not have directly mentioned recommendation algorithms but nonetheless discuss recommendation in some form, whether it be through an interface or an application. Including the term recommendation also tended to focus 


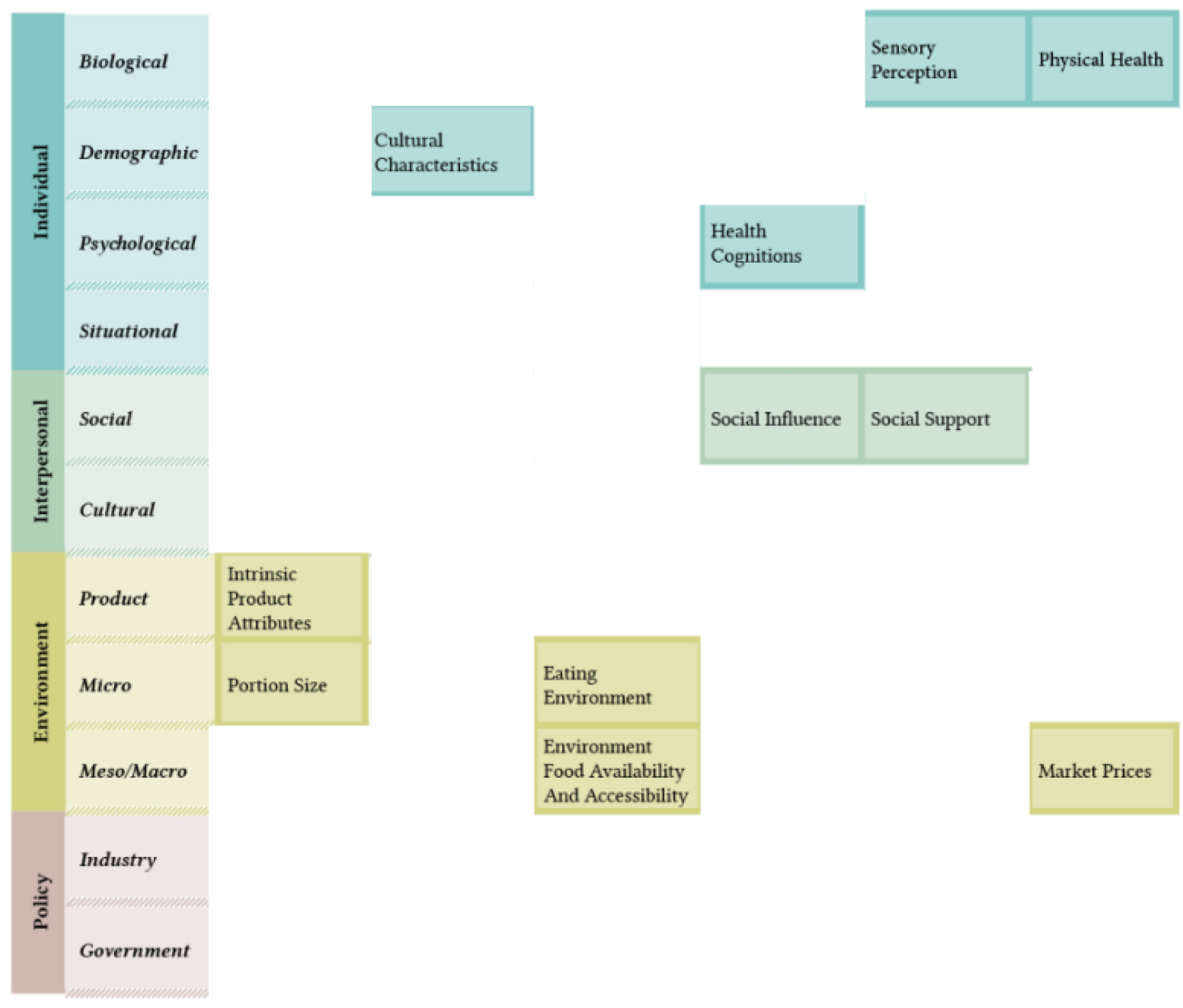

Fig. 2. DONE framework suggested by Stok et al. [62]. It depicts determinants of nutrition and eating behavior. The highlighted darker-colored groups (Sensory Perception, Physical Health, Cultural Characteristics, Health Cognitions, Social Influence and Support, Intrinsic Product Attributes, Portion Size, Eating Environment, Environment Food Availability and Accessibility, and Market Prices) represent the determinants identified as themes among the analyzed papers. The blank spaces are there to show the determinants that were not included in our research, as we focus only on those specific to this review.

heavily on technically related papers and, while we wanted to retrieve papers that used techniques such as machine learning, we did not have the intention to review only that area of research. For all of the searches, we used the same set of keywords with the exception of the IEEE database, for which these terms were too broad and included a vast amount of unnecessary articles. The latter was searched by using the words food, health, culture as words that were required to appear in the metadata along with any of the following: taste, nutrients.

\subsection{Filtering: The Exclusion and Inclusion Process}

The search resulted in 719 papers total, with additional papers found through forward and backward searches utilizing Google Scholar. The search was conducted by two of the authors. Inter-rater reliability was calculated on a voting basis. Once a paper was found that may be useful it was voted on by both authors. If both agreed, the paper was added to the review; if either disagreed, it was not added. 
2.4.1 Exclusion. The authors then filtered out papers that were not relevant to the criteria mentioned or did not include any comments related to health. For example, papers that discussed taste or recipe without discussing how it is related to the healthiness of the consumer were not included because our goal is to look at healthiness aspects, such as dietary needs. We also did not include image recognition papers that focus on recognizing various foods from images alone, as this is not related to healthy recommendations or healthy food influences. We also did not consider entries that contained only abstracts or were short panel papers. Papers were also removed if they were not directly related to the DONE Framework determinants, namely, Sensory Perception, Physical Health, Cultural Characteristics, Health Cognitions, Social Influence and Support, Intrinsic Product Attributes, Portion Size, Eating Environment, Environment Food Availability and Accessibility, and Market Prices.

2.4.2 Inclusion. Special care was taken to select specific determinants that would be used as inclusion criteria for the papers. The determinants selected were those that were most relevant to the review based on a vote by the authors. These determinants also happen to cover the largest amount of papers based on our search criteria, though this was not a basis for selection. The study also must have been published in the last 15 years, as food literature from a technological standpoint can be traced as far as the early 2000s based on the papers found in the search criteria. This narrowed the list of papers down to 76 in total. However, we wanted only those papers that focused specifically on food choice technologies in the review; thus, we ended up with 27 . In addition, we split the papers into two categories. One category was for papers that were correlation/study based only. These papers were required to consider factors that could be directly related to food consumption or influence eating habits. The other category was for papers that either presented or had in development healthy food choice technologies.

\subsection{Coding Criteria}

Last, we decided to use axial coding as the coding procedure. This allowed us to break the papers down and map them into three distinct themes that covered the entirety of the papers in this review. The themes are personal-related factors, environmental factors, and food properties. These are also in line with the categories that researchers in the consumer research discipline frequently suggest as the factors that determine food consumption [40,61, 63], Figure 1(a) depicts the categories/themes and Figure 1(b) shows the counts of papers in each category and any intersection among all three. This shows us how various researchers have appropriately considered these factors when devising their technological solutions. However, few have considered the combination of factors concerning all three categories.

\section{OVERVIEW OF THE SELECTED PAPERS}

Interest in the use of technology specifically designed to support healthy food choices has increased over the years, with a decline occurring only in 2015 to 2016. Table 1 shows the publications by context that specifically focus on a technology that has already been developed to support healthy food choices. The earliest work included in this review was published in 2003. The final count of the main set of papers included in this review that specifically cover the use of technology designed to support healthy food choices is 27 , which are distributed in 22 different publication venues, including CHI, Ubicomp, and RecSys.

\section{FINDINGS}

In the following sections, we present examples from the literature, placing them in our survey framework.

\subsection{Categories of Factors Considered in Healthy Food Choice Technologies - RQ1}

In this section, we discuss the three common themes that were found among the papers: (1) person-related factors, (2) properties of the food, and (3) environmental factors. For each theme, we discuss determinants of the DONE framework. We chose these specific determinants (i.e., choosing to discuss cultural over social) because 
Table 1. Count of Papers with Developed Technology Included in the Analysis

\begin{tabular}{c|l}
\hline Context & \multicolumn{1}{c}{ Author } \\
\hline \multirow{3}{*}{ Grocery (8 papers) } & Shekar et al. [58] \\
& Wu and Teng [80] \\
& Reitberger et al. [50], Reitburger et al. [51] \\
& Ahn et al. [5], Wayman and Madhvanath [75] \\
& Adaji and Vassileva [2], Siawsolit et al. [59] \\
\hline & Grimes et al. [33] \\
& Ueta et al. [71] \\
& Chen et al. [11] \\
& Almonani et al. [8], Chang et al. [9] \\
& Elsweiler et al. [22], Ge et al. [30], Lo et al. [41], Park et al. [48] \\
& Chaudry et al. [10], Forster et al. [24], Ueda et al. [2016] \\
& Agapito et al. [3], Min et al. [44], Trattner et al. [70], Yang et al. [81] \\
\hline Personal (16 papers & Ntalaperas et al. [47], Takeuchi et al. [64], Zhang et al. [85] \\
\hline Social (3 papers) & 27 papers \\
\hline
\end{tabular}

the papers in this space covered those determinants more directly and we wanted to discuss less-covered areas of research here, such as how culture affects food habits.

(1) Person-Related Factors. In their efforts to aid healthy food consumption, researchers have frequently relied on user preferences along with different dimensions of the user's characteristics, such as biological (e.g., sensory perception and physical health), demographic (e.g., cultural characteristics), psychological (e.g., health cognitions, food habits, and social influence and support), and situational (e.g., situational and time constraints) [62].

- Sensory perception refers to food taste preferences and aversions. It can be a useful factor in influencing food choice since studies have shown that consumers are more likely to eat food that they enjoy sometimes regardless of anticipated unhealthy consequences. For example, Forwood et al. [25] conducted a study that manipulated labeling to examine the effects of sensory and health information embedded in the food label. In their study, they changed the labeling of an "apple" to "succulent and healthy apple" and found that $15 \%$ more participants chose the apple over candy bars when labeled as "succulent and healthy." This study showed how important implied taste of a product can be for the user regardless of healthiness and that the taste factor should be strongly considered. To further confirm that taste plays an important role when choosing food, Freyne and Berkovsky [27], and Ge et al. [29] developed recommendation systems that considered the user's ratings (taste preference) at the ingredient level. Ge et al. also considered user preferences and weight goals for recommending recipes.

- Health cognitions refers to the person's health consciousness and healthy eating motivations. Almonani et al. [8] developed a game known as MACO that aids in teaching children healthy eating habits and introduces fun activities to encourage them to exercise more. The game also informs children about the harm of eating unhealthy foods. The goal of this game was to lower childhood obesity rates.

- Physical health can be affected by diet. To ameliorate the consequences of eating habits on dietary diseases, Agapito et al. [3] and Lo et al. [41] developed personalized dietary systems to provide food recommendations to aid those who have chronic dietary diseases, such as Chronic Kidney Disease (CKD), hypertension, and diabetes. To provide these recommendations, user profiles were used as features of the system and foods were recommended based on regional choices and health condition. Ahn et al. [5] developed an augmented reality (AR)-assisted mobile grocery-shopping app to influence shoppers in grocery stores to buy healthy items. The system provided the users the dietary constraint labels along with 
nutrition information. Similarly, Shekar et al. [58] developed a mobile app that assisted grocery shopping by recommending products that match the user's medical condition indicated in the user's profile. Ueta et al. [71] developed a goal-oriented recommendation system that utilized an interface allowing the user to type in information, such as searching "I have a cold" and a list of recipes would appear that could address the searched condition.

- Cultural characteristics such as cultural cognition (i.e., cultural norms), cultural behaviors (i.e., cultural eating habits), and cultural characteristics (i.e., ethnicity) have been identified as factors that influence food consumption. However, only a few researchers have considered them in their proposed food choice technologies. Cultural characteristics refer to the thoughts, behaviors, and beliefs related to one's cultural background. Al-Nazer and Helmy [7] utilized an ontology encased with cultural variables such as religion, place of origin, and language along with user health profiles to improve semantic interpretation (characteristics/demographics) of the user. Thus, they were able to better match users with their food interests.

- Social influence and social support refers to diet and eating-related influences and support from others in the environment [62]. Chang et al. [9] developed "Lunch Line," a system that relies on social influence in the workplace. Researchers used "public displays and personal mobile devices to encourage healthier eating" by allowing the user to see what foods others were eating at the cafeteria and how their own personal choices match the USDA recommendations. This project "was effective in 'attracting attention from the public, enabling self-reflection on eating habits, as well as giving the individual feedback on both group and personal choices"' [9]. Reitberger et al. [50] developed a system known as "Nutriflect" that encouraged healthier shopping habits in households. An interface was left in the kitchen to show families information summarizing their eating habit patterns as compared with the food pyramid or personal goals. This encouraged reflection, conversations, and positive changes in the household's eating habits.

(2) Properties of the Food. According to Symmank et al. [63], the properties of the food has been the most widely studied factor regarding food choice. Our analysis also shows that this category is common when considering dietary concerns for meal plan recommendation algorithms $[69,81]$ or when comparing against guidelines [9]. The papers included in this survey have mainly focused on the flavor and nutritious composition of the food.

- Nutritious composition, an intrinsic attribute of the food, has been studied in different contexts. Elsweiler et al. investigated "how people understand and choose recipes online to determine how feasible it would be to replace online recipes deemed unhealthy with those deemed to be healthy" [23]. Their study suggests that the recommendation process can be manipulated such that consumers may choose a healthier recipe more often. The research by Elsweiler et al. [23] examined how consumers cannot effectively distinguish between recipes that have significantly more fat than another similar recipes when relying only on images. Then, they developed a food recommendation system that considered low-level images and recipe metadata such as the amount of sugar, sodium, fat, and saturated fat as predictors to find suitable alternative recipes. Ahn et al. [5] proposed a mobile-based AR system to assist shoppers in grocery store aisles in choosing healthy items. The system presented the user's tags (dietary constraint labels) along with nutrition composition. Other researchers also explored combining health concern variables (i.e., restraints such as low sodium) and nutritious composition to assist selection of food in the context of grocery shopping [58,59] and restaurants [47]. Wayman and Madhvanath [75] created "Foodle," an interface that presented users with goal-driven food recommendations after analyzing the product list gathered from the grocery receipts. The recommendations are based on the gap in nutrition between the current purchase and a nutrition target. Similarly, Reitberger et al. [50,51] utilized grocery shopping receipts to create a visualization that displayed a food pyramid and nutrition information of the purchased items. This fosters reflection about consumer shopping habits, which aids families in adopting healthier foods choices. Also, Forster et al. [24] developed a nutrition dietary feedback system 
for steady and personalized dietary advice to consumers by allowing them to select their own nutrition preferences. Smaller amounts of saturated fatty acid and salt and higher amounts of dietary fiber were the most commonly selected consumer goals. The feedback system was built using an embedded ranking method, decision trees, and personal feedback reports. Results showed that the automated feedback system was on par with the manual feedback system, which suggests that automated systems can be utilized for educating and influencing the consumer on the benefits of a nutritious diet.

(3) Environmental Factors. This category comprises micro and macro variables related to consumers, food, and their environment. Stok et al. [62] define micro variables as being influenced by people (e.g., food portion sizes and eating environment). They describe macro variables as being related to the consumers' surroundings, which may be beyond their control (e.g., environment availability, food accessibility, market prices, and characteristics of living area).

- Portion size is the factor that has been more frequently studied as an environmental micro variable. Food portion sizes have been considered in situations in which patients with obesity need assistance with learning how to limit their food portion size [10], in which food intake is monitored to alleviate malnutrition [15], or in which assistance is provided to automatize food identification and intake monitoring that could help to achieve dieting goals [11, 45].

- Environment food availability, accessibility, and market prices refer to geographic location and socioeconomic status of a community, which can influence the access and cost of healthy food [62]. Wu and Teng "proposed a recommendation technique for online grocery shopping" [80] that considered the economic factor. This technique used three factors: "individual interests, product replenishment and product promotion, to suggest a personalized grocery shopping list" [80]. Similarly, Shekar et al. [58] developed "iGrocer," a personalized grocery shopping assistant that kept track of users' expense history and advised them "on what products to buy and what to avoid based on nutrition criteria and price constraints" [58].

- Eating environment refers to the social/personal surroundings of the consumer. Takeuchi et al. [64] considered users' meal satisfaction/preferences and eating environment to improve the user's eating habits. Researchers proposed a social media-based system that adapts users' meal evaluation to factors considered important by the user. The six factors used to determine meal satisfaction were taste, healthiness, human environment, dining environment, fashion/gourmet, and economy. The researchers influenced the participants to eat healthier by manipulating the preferences of the participant's social group to reflect healthier choices.

4.1.1 Discussion: Gaps and Opportunities for Factors Considered in Healthy Food Choice Technologies. While there are many studies that discussed the development of food choice technology, most did not address factors such as cultural food habits and environmental factors, such as being located in a food desert. Many of the factors we will discuss in this section have been noted in the DONE Framework [62] as important, yet researchers are not using many of these variables in their food choice technologies. In this section, we discuss how some of these additional factors may be included in food choice technology design.

(1) Person-Related Factors is an area that could be researched further.

- Cultural Characteristics have been considered in several studies that were intended not to develop a tool or system but rather to understand the correlation between cultural factors and eating habits. For example, Hormes and Niemiec [36] carried out a study to see whether the culture surrounding women in the United States was correlated with craving chocolate during the menstrual cycle. This was shown to be true, as women in other nations did not show a cultural craving during menstruation. Sajadmanesh et al. [55] performed a recipe-centric study to understand how cuisines differ worldwide and how the consumption of those cuisines correlates with the health statistics of that country. The purpose of the research was to aid in identifying foods that users are more likely to consume based on country and any potential unhealthy consequences associated with them. Habits and routines around food consumption 
also proved useful in building food recommendation models. For example, Silva et al. [60] developed a clustering model based on what users eat and when they eat to discover cultural boundaries across geographic regions. While this effort uses consumption to predict cultural boundaries, it nonetheless demonstrates how models can integrate information around culture and food. These examples from the literature demonstrate how cultural information can and should be used in the design of food choice technology in order to make more appropriate and useful recommendations for users based on their specific cultural context.

- Food Knowledge, Skills, and Abilities, as noted by the DONE framework, are important determinants of food choice and potential opportunities for research. They refer to "personal resources that are relevant to dieting and eating" [62]. Food and nutrition knowledge, for example, were studied by Forwood et al. [25], who concluded that food labels that appeal to the consumer can lead to them choosing unhealthy foods over healthier foods even if they are aware of which is healthier. Other factors, however, have not been considered. For example, it may also be important to include cooking skills since they can greatly influence what one is comfortable preparing for a meal. However, this has not been proven in the literature of the reviewed papers.

- Family Structure and Family Food Culture describes the composition and cohesion of the family household as well as the food culture existing in the family/household [62]. Variables such as household size, food processing methods, and family preferences were rarely mentioned in any of the papers with regard to how they may influence a consumer's eating habits. In fact, only Reitberger et al. [51] looked into family food culture to aid in healthier grocery shopping habits for the household. More such work can potentially uncover how to leverage family structure and family food culture to better design technology for food choice.

- Physical health-related variables were also considered by some of the research efforts to understand the indicators of disease. West et al. [76] performed a web-based study to investigate links between recipes' sodium content and hospital admissions for heart disease. The analysis considered population-wide data over time and regions. This study found a correlation between increases in sodium intake and hospital admissions over time. Sodium intake and region in which the user lives may be factors that should be considered. Mejova et al. [43] utilized the social networks Foursquare and Instagram to assess the link between fast food restaurants and obesity. This data-driven approach shows how fast food restaurants' social media favor unhealthy food choices, which may lead to dietary-related diseases such as obesity. Thus, social media-related variables may be a big factor, though more research on this topic is needed to develop concrete results.

- Social influence and social support variables related to food choice and habits have been studied by various researchers. Yet, these variables are rarely implemented in any food recommendation systems. Chung et al. [14] studied how people use Instagram to track their food intake and to receive social support that helps them reach their health goals. Cunha et al. [17] reported on the positive influences of the social networking platform Reddit for weight loss and to combat obesity. Results of the study showed an increase in overall likelihood to lose weight by $26 \%$ if social support was received on their post. Whether the user received support or not determined whether that user was likely to return to the Reddit forum. On the other hand, Mejova et al. [43], using a data-driven approach, revealed that social platforms could also negatively influence food choices. After analyzing Instagram and Foursquare data, it was inferred that social approval -e.g., likes - often favor unhealthy food choices and that popular small businesses were linked to obesity. This suggests that social variables can and potentially should be utilized as user features in machine learning models around food choice, habits, and behavior and in relation to interface design.

- Situational and time constraints affect food consumption [62] and were studied by Wagner et al. [73]. By analyzing online food preferences, search of online recipes by people, they found that regions that are closer together share similar online food preferences. Also, regional food preferences differed 
on the weekend as compared with weekdays. For example, according to the study, foods (cheap and healthy) rich in carbohydrates are consumed more at the beginning of the week and taper off on the weekends. This is just one example of how time can be leveraged to potentially make more effective recommendations. Or consider, for example, how recommendations might differ for someone in a hurry who only has 20 minutes to prepare a meal versus someone who has one hour to prepare a meal. Can we leverage this constraint information to make more effective recommendations?

(2) Environmental-Related Factors such as accessibility, living area, and food labels are all related to food choice and should be further investigated.

- Environment food availability, accessibility, and market prices, as mentioned before, can influence the access that consumers have to healthy food. The technical community has mostly focused on studying social network information to understand environmental characteristics of consumers $[1,10,16,32]$. However, nutritionists and researchers in the food sciences have studied this topic in more depth $[31,35]$ and their findings should be more tightly integrated with the technical community's approaches. Gordon [31] studied the effects of environmental exposures, such as limited neighborhood access to healthy foods, on health outcomes in various races, regions, and urbanicities. Herforth et al. [35] also studied environmental effects on health, such as the link between income level and healthier diets. They also investigated the effects of the food environment, such as agricultural interventions and limitations of food availability, in certain areas. From the social network perspective, Choudhury et al. [13] used social media to show that people in low-income areas post more about foods high in fat and that living in food deserts can vastly impact the types of nutrition that people have access to. Gore et al. [32] utilized Twitter and geographic location to identify how people in areas with lower obesity rates in general post happier tweets and frequently discuss exercise, fruits, and veggies. Culotta [16] utilized Twitter data in order to investigate whether health concerns can be inferred across the nation. The study covered " 27 healthrelated statistics including teen birth rates, obesity, health insurance coverage, and access to healthy foods" [16]. The results of the model were strongly correlated with lack of insurance, vehicle mortality, teen birth rate, limited healthy food access, dental access, and obesity. These studies provide ample environmental factors for consideration and, when combined with what is already known in the nutrition and food sciences, could lead to future research on how to design technology to prevent environmental disadvantage for consumers who may not have access to or cannot afford healthier food options.

- Characteristics of living area were also pointed out in the work of Cheng et al. [12], who helped provide insight on another characteristic of living area to consider, city size, when making food recommendations. City size can play a major role in what users decide to eat, as the eating habits of those who live in urban and rural areas can vastly differ. Cheng et al. developed a recommendation model that considers time, location, and city size. They found that people in bigger cities tend to eat more food from other regions while smaller cities tend to eat traditional food related to their specific region/culture.

The DONE framework provides importance scores for various factors of food choice. Many of the highest scored factors are environmental-related variables, such as healthy food availability. Without access to healthy foods, consumers may be less driven to eat healthier due to inconvenience. Distance to supermarkets and healthy food sources could be important for recommendation systems and interfaces that are tailored to support healthy food choice. We suggest that future recommendation models consider these as well as the other environment factors (i.e., distance to grocery stores) mentioned earlier.

\subsection{Context of use - RQ2}

Smart devices that can be carried everywhere, connect to the Internet, and facilitate location-based services have made it possible to implement food choice technologies in different contexts. In this section, we discuss three contexts in which food choice technologies have been explored: grocery shopping, social, and personal. 
(1) Grocery shopping. Grocery shopping is a major part of household activities and typically the main avenue for average consumers to attain food for themselves and their families. Therefore, consumers should be mindful while grocery shopping to consider nutrition, dietary restrictions, and dietary-related diseases. Researchers have explored the grocery shopping context in a variety of ways. Shekar et al. [58] developed "iGrocer," which is an application to aid users in finding their food items quickly in a grocery store while taking into consideration users' medical condition, food nutrition content, and price constraints. It also included a rewards strategy to influence the customer to buy healthier foods. From the study by Shekar et al., we learned that in the grocery context, a rewards system can influence how consumers shop for groceries. Reitberger et al. [51] developed the "Nutriflect mobile," a tool that provided situated visual aids for improving customers' nutrition practices by comparing their selected products against the standard food pyramid's recommendations for daily consumption and the consumer's personal goals. It also informs the research community about the consumers' grocery shopping patterns, in particular, their family's patterns, in this context. Ahn et al. [5] utilized AR on a mobile device to instantly augment the product information on the shelf. Once a product is identified, the AR app displays a healthy/unhealthy color-coded tag, product name, product description, and its nutrition label. This expedited the process of finding healthy food, decreasing the time to search for foods by 2 to 3 times. The AR app also provided healthy recommendations through a matching process based on the user's health preferences and dietary guidelines.

This suggests that consumers may benefit from having a tool that can display a food's nutritional value as the consumers that were included in the study generally commented that they were "Highly Satisfied" with the application. Other researchers suggested reflection tools that employed grocery shopping receipts data. Wayman and Madhvanath [75] developed "Foodle," a system that used as input the user's grocery receipt data to provide food recommendations that follow the user's dietary guidelines. Foodle also provides personalized visualizations that allow users to track and see their current state of nutrition consumption and compare them with their dietary goals. Foodle provided researchers with insights that potentially prove how important sufficient grocery information can be for personalized and family food recommendations. These systems (iGrocer, Nutriflect Mobile, Foodle, and the AR Mobile system) are examples of approaches that engage consumers in buying healthy food by allowing them to see nutrition content and compare them against personal goals or health standards, which ultimately could invite consumers to reflect on their food habits.

On the other hand, research [39] has shown that although consumers care about health and price when buying products, they are frequently overwhelmed by technologies that assist shoppers when the technologies present too much information meant to support their decision-making. To support people's "fast and frugal" shopping behavior, recommendation systems come into play. Wu et al. [80] developed a recommendation scheme that considers purchase history of the consumer along with product replenishment $-\mathrm{a}$ constraint that indicates the unlikelihood of buying the same item in a short time span, price, and product promotion - to consider the buyer's budget. This recommendation system, instead of assisting the user during the selection of items, presents the user with a suggestion for a grocery shopping list.

(2) Social Context. The social context describes how consumers interact with the social environment around them and how that influences eating behaviors. Food choice technologies have been developed to serve the social context, such as bars and restaurants, parties, and schools. Prior research has already shown that consumers are positively or negatively influenced by the restaurants they frequently visit [38]. With this realization, systems were developed to aid in restaurant recommendations. Ntalaperas et al. [47] developed a system known as DISYS for aiding users in choosing restaurants that fit their dietary situation, such as trying to eat healthily or trying to eat particular foods due to a dietary condition (e.g., obesity). DISYS gives researchers understanding into how consumers can still eat healthy even within a social setting such as eating out with friends. Social media can also be considered a social context, as it can influence users' eating habits [57]. Zhang et al. [85] also developed a restaurant recommendation system for recommending 
the next best restaurant to the user based on prior restaurant visits and demographic factors. While the study by Zhang et al. may not focus on the health aspect specifically, it does provide us with insights on how to recommend future restaurants to consumers that may fit criteria of prior restaurant visits within this context. Takeuchi et al. [64] considered feedback from other users regarding the dining environment, budget, and meal satisfaction when suggesting food choices. This work gives us details on how a feedback mechanism within social-based applications can aid in meal satisfaction for the consumer while still considering healthiness of the meal.

Additionally, studies were developed that focus on the social context but did not directly involve system development. A particular Reddit study found that when consumers have support groups on social networks, they achieve their weight loss goals at a higher rate, which demonstrates the effects of having a social support system [17]. Rokicki et al. [54] performed an analysis that showed how user feedback, such as ratings and comment behavior, is influenced by the recipe author's reputation, friendship relations, similarity between user profiles, and temporal and seasonal effects. One could suggest that this analysis shows how crucial the social aspect is with regard to popularity for an online recipe. Predictive models were developed to predict mean sentiment, number of ratings, and number of views based on various social features such as comments by friends, popularity features such as number of ratings, temporal features such as season, editorial features such as recommended recipe, and gender features such as male ratings. Ye and Lee [83] proposed a project that utilizes mobile technology for gathering smartphone usage data from an undergraduate freshman population in order to construct activity patterns and behavioral changes related to weight gain. The goal was to understand social spaces by discovering correlations in behavioral changes and health outcomes, with the motivation being to limit increasing cases of obesity. In the data analysis step, the researchers planned to investigate how social networking with peers, levels of physical activity, and frequent visits to various food outlets may affect weight changes. From these studies, we can discern that social context can have both positive and negative effects. Therefore, it may be important to bias users towards healthier foods and approach the topic of healthy food decision influences through technology from different contexts.

(3) Personal Context. The personal context considers how consumers may be influenced to have certain eating habits based on their own personal values and personal usage/tracking of applications. Most commonly, food choice technologies that focus on the personal context are interfaces that consider: (1) users' profile for health preferences or disease-related recommendations [3, 24, 41, 47, 71, 81], (2) users' food preferences (likes or dislikes) for healthy recipe or meal plan recommendations [27, 69, 81], (3) aids to identify and track users' food intake [10,11,15, 45], or (4) educating users about healthy habits [8]. Research has shown how the personalities/characteristics of users can aid in improving recommendation agents for healthier foods. The evaluation by Elsweiler et al. [23] showed that consumers, given the choice of two dishes, will likely choose the dish containing the highest amount of fats without consciously knowing if one has more than the other. Therefore, a machine learning model was developed to provide replacement recipes to users based on features that included user preferences. The way users interact with an interface can also be seen as the personal context as this determines how they may or may not use the information learned from the app. Ge et al. [29] were able to develop a health-aware recommendation system with a user interface that took the user's profile (calories goal) into consideration when recommending healthy recipes based on the ingredients the user likes. The interface depended heavily on a single user input for information and tracked any changes made to the profile over time. The study by Ge et al. helps to inform researchers on the scope of recommending healthier recipes to consumers that are cooking at home. Trattner et al. [70] also performed a data-driven study and utilized personal hobbies such as biking and knitting to see how they correlated with a consumer's nutrition values. Particular hobbies such as fishing and hunting were associated with higher intake of proteins while others such as knitting were associated with higher fat and 

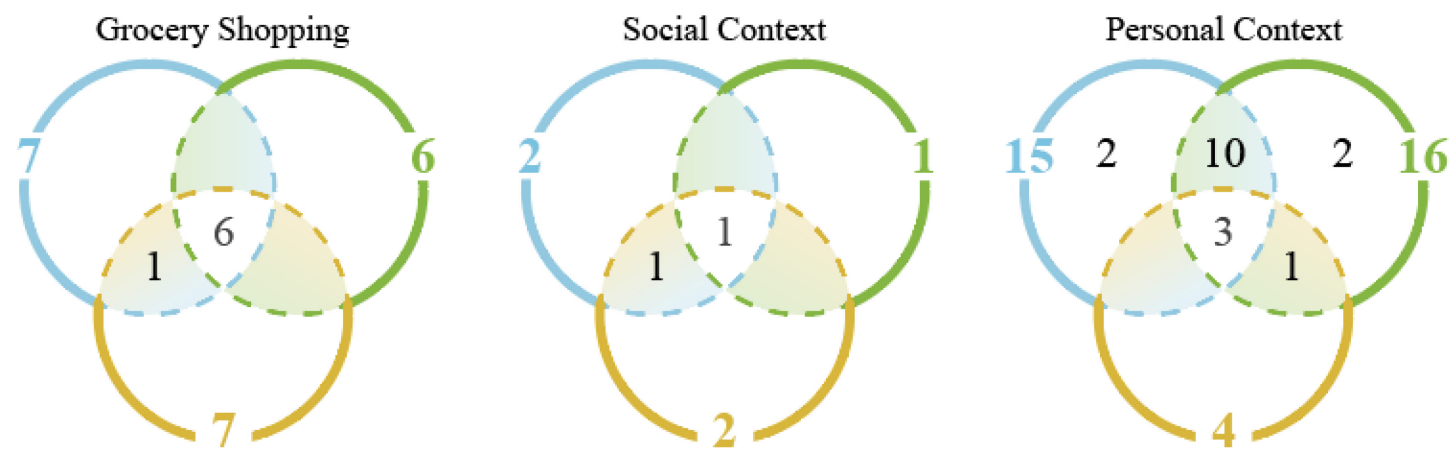

$\square$ Person-related Factors $\square$ Properties of the Food Environmental Factors

Fig. 3. Set of papers grouped by context of use and categories of factors.

carbohydrate intake. This provides researchers with details on how the habits of the user may affect food consumption within this personal context

4.2.1 Insights Gained from Context of Use. While the chosen papers for this review were fairly extensive in covering target use cases (i.e., grocery shopping, social, or personal context), we were able to identify a particular gap within the context of use. Figure 3 displays the chosen papers grouped by the context of use and factors. We can see that more research is needed within the social context while the personal context researchers should consider more environmental variables in their studies to provide a better experience for the consumer. Also, based on our analysis of the papers within these contexts, we concluded the following. The grocery context benefits greatly from applications that provide some form of reward for healthier shopping and from applications that display health information of foods to the user. The social context benefits from applications that can aid users in choosing restaurants that fit their dietary needs. The personal context can benefit from applications that focus solely on providing a single user with recipe recommendations that suit the user's particular need only and are more personalized in nature.

\subsection{Technological Approaches to Healthy Food Recommendation - RQ3}

Researchers have taken a variety of technological approaches in order to influence consumers to choose healthier eating options (Table 2). In this section, we discuss approaches that have proven most useful, such as classification models [22, 24, 65, 71], recommendation systems [3, 30, 41, 47, 69], social network platforms [17, 64], and other interfaces/apps [5, 7, 8, 15, 33, 45, 48, 49, 75]. Additionally, we identified in which context of use each technological approach works best.

(1) Classification Models. A classification model is a supervised machine learning approach that makes predictions on a target variable from observed values. In the context of healthy food choice technology, classification models have been utilized for performing specific tasks, such as generating meal plans for users and aiding in predicting various tastes, such as sweet and salty, that users may enjoy. This includes the work of Ueta et al. [71], in which a food goal-oriented classification system was developed to aid users in selecting healthy foods depending on their health condition. For their approach, data were collected on diseases and foods/nutrients that have been known to aid in addressing the diseases, such as pantothenic acid aiding in the treatment of acne. Elsweiler et al. [22] developed a nutrient-based classification system as well as a recommendation system for generating meal plans based on 100 user profiles combined with food preference data. They collected user ratings of recipes and user demographic data to build linear models for predicting the various personas of users, as personas were indicative of what users may eat. Forster et al. [24] 
Table 2. Overview of the Technological Approaches Used in the Papers and Context of use they Work Best

\begin{tabular}{|c|c|c|}
\hline Technological Approach & Representative Work & Context \\
\hline \multicolumn{3}{|l|}{ Classification Models } \\
\hline + Supervised Learning & $\begin{array}{l}\text { Ueta et al. [71], Teng et al. [65], Forster et al. [24], } \\
\text { Elsweiler et al. [23] }\end{array}$ & Personal \\
\hline \multicolumn{3}{|l|}{ Recommendation Systems } \\
\hline + Collaborative Filtering & Trattner et al. [70] & Personal \\
\hline + Content Based & $\begin{array}{l}\text { Ge et al. [30], Ntalaperas et al. [47], Agapito et al. } \\
\text { [3], Yang et al. [81] }\end{array}$ & Social/Personal \\
\hline + Hybrid Based & $\begin{array}{l}\text { Freyne and Berkovsky [27], Wu and Teng [80], Lo } \\
\text { et al. [41], Zhang et al. [85] }\end{array}$ & Social \\
\hline + Knowledge Based & Takeuchi et al. [64] & Social \\
\hline \multicolumn{3}{|l|}{ Social Network } \\
\hline + Social Feedback & Takeuchi et al. [64] & Social \\
\hline + Social Support & Cunha et al. [17] & Social \\
\hline \multicolumn{3}{|l|}{$\begin{array}{l}\text { Other Types of Technological } \\
\text { Innovations }\end{array}$} \\
\hline + Food Journaling & Comber et al. [15], Choudhury et al. [13] & Personal \\
\hline + Grocery Apps & $\begin{array}{l}\text { Kalnikaité et al. [39], Reitburger et al. [50], Ahn } \\
\text { et al. [5], Siawsolit et al. [59] }\end{array}$ & Grocery \\
\hline $\begin{array}{l}\text { + Other Technologies (Semantic } \\
\text { Search and Information Visualizations) }\end{array}$ & Al-Nazar and Helmy [7], Chang et al. [9] & Personal \\
\hline
\end{tabular}

developed a dietary feedback system to deliver personal dietary advice utilizing decision trees for prediction of the type of advice to be delivered. The advice to be delivered was based on the nutrient intake, such as saturated fatty acid, protein, salt, and dietary fiber, as those were used as features for the prediction model. The advice was broken into four levels, with higher levels indicating that more health-related advice was needed based on the user's current nutrient consumption. An example of an advice message recommended by the algorithm is "Eat more fruits and vegetables - aim for at least a day." Teng et al. [65] developed a recipe network and utilized stochastic gradient boosted trees to aid in predicting what users would rate a recipe before it was rated. The goal was to discover ingredient complements and ingredient supplements for foods with future hopes of utilizing the network for predicting diet-specific ratings. Notably, particular features that have been utilized by classification models are demographic information and the nutrition content of foods. Classification models are useful for predicting overall characteristics about consumers and recipes, such as nationality, or whether a particular recipe is healthier than the other but typically do not perform better than recommendation models such as collaborative filtering due to their inability to provide quality personalization to the consumer. However, they do often require less data to get a complete model than a standard recommendation system.

(2) Recommendation Models. Recommendation models are a machine learning approach that utilize techniques such as collaborative filtering and content-based filtering to provide users with quality recommendations on particular items (e.g., AllRecipes.com). Traditionally, recommendation models have been used for recommending items such as books and movies based on a user's preferences. To date, there are limited research articles that focus on healthy food-related applications for recommendation models, though there 
have been several literature reviews published [67,68]. Variables that have been proven to be useful for food recommendations include user preferences in the form of recipe ratings and taste variables such as whether the user enjoys sweet or salty foods. They provide advantages such as dynamic learning and strong personalization.

(a) Collaborative Filtering. Collaborative filtering takes into account the similarity of interests between users to make predictions on what the current user would like. Trattner and Elsweiler [69] proposed a post-filtering method for algorithms such as single-item recommendation in order to develop healthconsiderate meal plans for users. While this method performed better than baseline recommendation approaches such as most popular item ranking, the author does state that this study could be improved by collecting more information about the user, such as taste preferences.

(b) Content Based. Content-based methods make recommendations for the user "based on items homogeneous to those the user has already liked" [30,47]. A particular content-based system, Yum-Me, specialized in personal recommendations for the user [81]. This system displayed a ranked list of foods based on learned preferences for the user to choose from. Agapito et al. [3] developed a content-based method using a system known as DIETOS to provide recommendations for patients with chronic dietary diseases based on their health profiles.

(c) Hybrid. Another method that has become popular is known as the "hybrid" recommendation model. Hybrid models combine aspects of collaborative filtering and content-based recommendation algorithms to achieve a new recommendation algorithm. Hybrid recommendations usually involve building on the weaknesses that a single model has on its own. For example, content-based approaches rely only on item descriptions for recommendations; thus, they are able to recommend foods to a user regardless of whether the system has data on past users or not. However, collaborative filtering may suffer from a lack of information on past users, as this approach relies on recommendations by utilizing the similar interests of other users. Freyne and Berkovsky [27] developed a hybrid recommendation model for food recommendations based on the scoring of the recipe ingredients as opposed to the recipes themselves. Zhang et al. [85] also utilized a hybrid recommendation model known as context-aware collaborative filtering in order to make predictions on new restaurants that a user was more likely to visit based on restaurant contextual features and user constraints such as age and sex. This model can be useful for health-based systems, as it gives information on what features may be important for restaurant recommendations and potentially healthier restaurant recommendations. Wu and Teng [80] utilized a hybrid recommendation model for online grocery shopping that incorporated product individual interests (purchasing habits of individual users), product replenishment (consumers buying the same product repeatedly), and product promotion (customer sensitivity to product promotion).

(d) Knowledge Based. Knowledge-based models focus on explicit knowledge about the user preferences and recommendation criteria (which items should be recommended in different contexts). Takeuchi et al., for example, considered the taste of users in order to improve eating habits [64]. These recommendation methods, while useful, often do not utilize user-centered research. Thus, a potential gap in this area is the limited use of real-world studies for evaluating recommendation models.

(3) Social Network Platforms. While social networking platforms have been studied in relation to consumer habits related to food, the findings from these studies have not been heavily utilized in food choice technologies. Social network platforms were used as a technique for gathering information to research people's preferences and habits [32] and for gathering feedback that would feed recommendations models [64]. Our analysis focused on social networking techniques that gather information on the users [32]. The purpose of using a social network approach is to gain more information about users that may otherwise have not been attainable, such as their eating habits, personality, and television habits, by searching their online social context. The term social context was emphasized in Section 4.2. However, in this case, we also find that the social context includes an online presence with social media. Most recommendation models built 
around social presence considered Twitter and/or Instagram data. From a systems standpoint, Takeuchi et al. [64] developed a social media-like platform to obtain feedback on user preferences for particular foods, which caused the dietary habits of the user to improve over the course of one month.

From an analysis of variables standpoint, Sharma and Choudhury [57] utilized Instagram to measure the types of food that people were frequently mentioning, which turned out to be the unhealthier ones, such as donuts. This was a correlation-based study to understand the types of food that were frequently mentioned on Instagram. Cunha et al. [17] utilized the social networking platform Reddit in order to capitalize on the understanding of group support within online spaces. The goal of their research on Reddit was to understand whether strong support for an individual to lose weight would motivate the person to continue losing weight. Studies suggest that more research should be done in this area to gain insights into user preferences that may not be gained through traditional approaches such as surveys. This lack of social network analysis may be a major gap in the healthy food decision-making space. Besides limited data, another downside of social network analysis is that people often report unreliable information in these settings and, thus, can easily skew analysis, as is reported in [74].

(4) Other Types of Technological Innovations. The process of using interfaces/apps to provide insight and guide users towards making healthy food choices or improving their food intake was a common approach for many of the chosen papers. This type of technological approach did not often use underlying technologies such as machine learning models. For example, some papers utilized AR to support users while doing grocery shopping [5], an ontology for recommendations based on cultural characteristics [7], and implemented mobile apps for tracking food intake and facilitating reflection and learning [15, 50]. The interface "Lunch Line" [9]was developed to persuade healthier eating at an information technology company. Public displays and mobile visualizations provided insightful information that facilitated users' reflections while comparing their own work-based cafeteria eating habits against their coworkers and USDA recommendations. Al-Nazer and Helmy [7] developed a semantic search application that relied on ontologies infused with user health profiles as well as their cultural and language characteristics. Their goal was to show how cultural aspects can improve food recommendations. Chaudhry et al. [10] developed an interface known as DIMA (Diet Tracking Mobile Application) that enabled users to record their meals using a photo. This is intended to help health-illiterate users and chronically ill people in their ability to measure food portion sizes. There were also various mobile apps for grocery shopping that aid users in selecting healthier food choices $[5,51,58,59]$. While this type of approach has a clear advantage of providing an interface to the user, they do not often employ some of the more advanced machine learning techniques utilized with the other methods. Thus, they can sometimes be limited in capabilities and useful recommendations.

4.3.1 Gaps and Opportunities in Technological Approaches. Upon review of the technological components of the gathered articles, we noticed several opportunities that researchers could take advantage of in order to produce more viable food choice technologies. One approach involves utilizing social networking data to make better recommendations. Social networking data can provide more insights about users that they may not realize themselves, such as temporal eating habits and how often they consume snacks/junk food. We can also use social networks to gain information about social influence and social support variables, such as the importance of friend/family dining habits, social norms, social relationships, community recommendations, and social support. Cunha et al. [17] showed how important social support variables were for maintaining weight loss goals. Thus, we conclude that social networks may need a more in-depth look while searching for factors that may influence food choices.

\subsection{Description of Dataset Types - RQ4}

Designing technological solutions that assist healthy food choices requires the combination of diverse datasets, including datasets that describe recipes, food nutrition content, the environment, and demographic 
Table 3. Dataset Reference Table

\begin{tabular}{|c|c|c|c|}
\hline Searching for & Datasets & Access Method & Differences in Dataset Loc. \\
\hline $\begin{array}{l}\text { Recipe ingredient } \\
\text { information, cuisine } \\
\text { attributes, taste } \\
\text { preferences, and user } \\
\text { ratings }\end{array}$ & $\begin{array}{l}\text { Allrecipes } \\
\text { Yummly } \\
\text { Epicurious } \\
\text { Kochbar } \\
\text { Cookpad }\end{array}$ & Web Crawler & $\begin{array}{l}\text { Cookpad and Epicurious } \\
\text { do not have taste } \\
\text { preferences. }\end{array}$ \\
\hline Dietary guidelines & $\begin{array}{l}\text { WHO } \\
\text { USDA } \\
\text { FSA }\end{array}$ & Publicly Available & $\begin{array}{l}\text { WHO has more macro } \\
\text { nutrient requirements } \\
\text { than FSA and rated } \\
\text { differently. }\end{array}$ \\
\hline Demographic variables & $\begin{array}{l}\text { U.S. Census Bureau } \\
\text { FFIEC } \\
\text { questionnaire/survey }\end{array}$ & Publicly Available & $\begin{array}{l}\text { U.S. Census and FFIEC } \\
\text { is down-loadable. }\end{array}$ \\
\hline User location preferences & Foursquare.com & $\begin{array}{l}\text { Publicly Available } \\
\text { API }\end{array}$ & None \\
\hline User physical activities & MyFitnessPal & $\begin{array}{l}\text { Must request API } \\
\text { access }\end{array}$ & None \\
\hline $\begin{array}{l}\text { Food hashtags or social } \\
\text { variables }\end{array}$ & $\begin{array}{l}\text { Twitter } \\
\text { Instagram } \\
\text { Reddit }\end{array}$ & $\begin{array}{l}\text { API } \\
\text { Must request } \\
\text { access } \\
\text { API }\end{array}$ & $\begin{array}{l}\text { News-SMS text content } \\
\text { Photos and videos } \\
\text { Community forum } \\
\text { content }\end{array}$ \\
\hline
\end{tabular}

characteristics (census information, social platforms, or tracking apps). In this section, we discuss the various data types and resources used in the chosen papers and give an overview of the data resources presented in Table 3.

(1) Recipes dataset. Gathering recipe information normally involves gathering recipe metadata and/or ratings from popular recipe websites. Through our review, we discovered that the most popular recipe datasets originated from "allrecipes.com." Other examples of popular recipe websites include "epicurious.com," "yummly.com," "meishij.com," "cookpad.org," and "kochbar.de." Currently, the majority of these sites have a free public API that can be utilized for crawling their database. Some, such as Yummly, require a payment depending on the amount of data the user would like to collect. Researchers [65, 69] have utilized allrecipes.com to gather recipe information such as ingredients, publication year, region, type of meal (breakfast, lunch, dinner), ratings, holidays associated with meals, and comments. Separately, Min et al. [44] utilized Yummly's API to collect recipe ingredients, images, and attributes of recipes.

Through the utilization of online recipe data, researchers can gain insights into user-related aspects such as ratings, comments, and bookmarks. For example, Trattner et al. [70] discovered that uploaded recipes tend to be healthier than bookmarked recipes by analyzing allrecipes.com data. Online recipe data does not normally provide information such as user health status, hobbies, and demographics. However, that information can be gathered through other methods, which will be discussed in the following section. Many of the papers included in this review employed recipe data specifically to make recommendations that would influence users to eat healthier.

(2) Food nutrition content dataset. To gather nutrition-related data, researchers can rely on the World Health Organization (WHO), the UK Food and Standards Agency (FSA), the Environmental Safety and Health Affairs (ESHA), and the United States Department of Agriculture (USDA) databases, where nutrition 
information is stored. While many of the papers used online recipe and social networking data, researchers such as Trattner et al. [69] combined online recipe data with data from the WHO and FSA traffic light system for labeling food in order to make recommendations. Federal databases from the USDA and others such as the WHO appear to be the standard for gathering reliable nutrition information. The ESHA nutrition database also contains nutrition information on approximately 40,000 food-related items.

(3) Environment and demographic characteristics dataset. For demographic variables, the U.S. Census Bureau is a quality source for data. For other socioeconomic variables, such as income level, the Federal Financial Institutions Examination Council (FFIEC) is a quality source. MyFitnessPal also has data, such as physical activities of the user [37]. These less common ways of extracting data are not typically the dominant approaches but are still important for many of the recommendation/influential systems.

(4) User-Related Variables. Social networking platforms are normally utilized for gathering user-related variables. Social network crawling involves the utilization of an open-sourced API to gather streaming data and saving that information into a database. Datasets extracted from social networking platforms as related to our search were mostly used for data-driven studies instead of system development. The datasets mentioned in this section are examples of those available upon request from the author. Platforms such as Twitter are useful for gathering more personal and social information about the user. Silva et al. [60] created a dataset consisting of roughly 4.7 million tweets that contained check-in data from Foursquare. Each check-in also contained information about the venue, such as its geographic location (i.e., latitude and longitude) and category (i.e., Arts and Entertainment, College and University, etc.) The check-in data was then split into subcategories of the original categories such as fast food check-ins, which had 410,592 check-ins. Data were not directly collected from Foursquare as Foursquare check-in information is not readily available to the public. Abbar et al. [1] crawled the Twitter streaming API for about 50 million tweets, of which roughly $890 \mathrm{~K}$ tweets from $400 \mathrm{~K}$ users were selected due to their geographic location being the United States. Keywords within the tweets were selected to match as many food-related keywords as possible, such as "pizza." A dataset was then formed by selecting the top 500 most popular food-related words and adding their nutrition information.

Among the social networking data, a common factor is the use of geographical locations to understand various aspects of user dietary habits as well as information relating to culture, happiness levels, and understanding of food deserts. The usage of variables discovered through social networking platforms has seen limited research. Thus, these variables could potentially be used as quality features for input to healthy food-based machine learning models and apps/interfaces.

4.4.1 Insights Gained from Dataset Choices. While there were many variations of datasets utilized by researchers to build their systems, we believe that combinations of these datasets would bring the most successful outcome for influencing food decisions through technology. If researchers utilized datasets that represented both social networking data and recipe website data, they could utilize more variables in their prediction models or interfaces. Ensemble methods of machine learning, for example, can be used for prediction on variables from various heterogeneous sources. As shown by Choudhury et al. [13], social networking data can be important for environmental variables such as identification of food deserts as well as food topics that people are most interested in, which is not something that can be readily found in recipe website data.

\subsection{Evaluating Food Recommendation Technologies - RQ5}

Recommendation systems are often accompanied by a framework of offline experimentation to determine what algorithms or system designs best suit the underlying task, such as in Gunawardana and Shani [34]. It is critical to develop an appropriate evaluation framework for the underlying task. The food recommendation systems present their own evaluation perspective - they can require both a user perspective on design and use of system and/or evaluating the performance of the underlying algorithms. To that end, in the subsequent discussion, we 
consider evaluation methods that involve a user-centered method to evaluate the food recommendation system use or interface and evaluation metrics that involve empirical evaluation of the algorithms vis-à-vis their output (recommendations).

(1) Methods - Survey/Interview. Application/interface-related technologies and designs are often evaluated with exit interviews/surveys and/or rating averages. These evaluation methods were used to understand factors such as user satisfaction, user understanding and learning, and ease of use of the interface. Grimes et al. [33] developed an exit survey and gave exit interviews to participants that played the OrderUp game to see whether the game raised awareness towards healthy eating. Kalnikaité et al. [39] utilized audio transcriptions and post-questions to evaluate the effectiveness of their approach. Users were voicerecorded while grocery shopping using the authors embedded information technology that instantly provided information on the nutritional value and price of foods that they wanted. Experts later checked on how satisfied they were by analyzing transcriptions from the recordings. Siawsolit et al. [59] developed a recommendation-like system for healthy grocery shopping and evaluated the system using a 5-star rating system for how well users enjoyed their recommendations. Chang et al. [9] performed a 3-week field study on the "Lunch Line" application and its users. There was an evaluation within the application to gauge user enjoyment and engagement through daily reporting. Surveys were also used to understand how users reacted to the "Lunch Line" system. Overall, the common methods used to assess interfaces were exit surveys and post-questionnaires, which were either incorporated into the interface or performed after use of the technology. Surveys and post-questionnaires are particularly useful for understanding user perceptions of effectiveness and user satisfaction. A weakness of surveys and exit interviews is that they may not accurately reflect the user's true experience.

(2) Metric - Performance and Correlation. The food technologies presented previously often employ classification correlation analysis algorithms that must be evaluated. These algorithms are normally evaluated using a combination of metrics, including Accuracy, Precision, and Pearson Correlation. Accuracy is best used when you need to predict the class for a balanced dataset, meaning having approximately a similar distribution among your class variables. Precision is appropriate when you have an imbalanced dataset but false negatives do not matter as much to your problem. Recall is important for imbalanced data and where false negatives are a much more important factor for your dataset. Pearson correlation is often used when you want to identify relationships between independent variables. For example, Forwood et al. [25] utilized logistic regression to predict what a user would choose to eat and employed accuracy as the evaluation metric. Culotta [16] used ridge regression in order to predict 27 health statistics using 180 linguistic variables from demographic areas across the United States. Culotta also employed the accuracy metric. Abbar et al. [1] also used the accuracy metric to evaluate a series of regression models to predict obesity and diabetes scores. Six models were considered: a model with demographic variables, one with food name variables, one with calorie variables, one with both food name and demographic variables, one with linguistic variables, and one with both linguistic and demographic variables combined. West et al. [76] utilized the Pearson correlation coefficient in order to test the hypothesis that behavioral data in weblogs can be employed to discover potential relationships in diet and acute health problems.

(3) Metric - Recommendation Performance Measures. The metrics used to evaluate recommendation systems approaches such as collaborative filtering include Mean Average Precision (MAP), Root Mean Squared Error (RMSE), Mean Absolute Error (MAE), and Normalized Discounted Cumulative Gain (NDCG). MAP represents the mean of the average precision scores that are gained for all queries separately and is most appropriate when predicting for queries. RMSE represents the sum of the square of the precision and square of the bias and is appropriate for regression-based models that can predict a variety of integers. MAE represents the deviation between predictions and actual output and is useful when the variance of the error is critical, such as in finance. Finally, NDCG represents the normalization of the measurement of usefulness 
of a document based on position in a list and is appropriate for ranking algorithms. Cheng et al. [12] utilized MAP to show the improvements of Bayesian personalized ranking over most popular item ranking performance. Each algorithm recommended items that were related to the type of user, such as a metropolitan person being recommended metropolitan-related recipes based on ratings of other users living in metropolitan areas. Trattner et al. [69] utilized both MAP and NDCG in order to measure the performance of recommendation algorithms such as User and Item-Based Collaborative Filtering, Weighted Matrix Factorization, Bayesian Personalized Ranking, Latent Dirichlet Allocation, and Association Rules. Freyne and Berkovsky [27] utilized MAE for recommending recipes based on recipe ingredients from other recipes. While these are just common metrics used for evaluating recommendation systems in the chosen papers, there are also other common metrics, such as Normalized Mean Average Error and Precision-Recall [34].

4.5.1 Gaps and Opportunities in Evaluation Methods and Metrics. A common theme in the recommendation system papers is that many of them do not employ evaluation with real users but rather often perform offline testing [34]. This could be a potential problem, as there are many issues to consider regarding user satisfaction with recommendations provided by the algorithm for meals, recipes, and so on. For example, when Trattner et al. [69] attempted to generate healthy user meal plans, they performed offline testing and mentioned this in the discussion section of the paper. Users should indeed be employed to evaluate systems that rely on user satisfaction in real-world use cases. One could also consider evaluating systems with recommendation explanations. If users are given an explanation as to why a particular food was chosen over the other, this may aid in their recommendation satisfaction and comfort with the application.

\section{DISCUSSION AND FUTURE DIRECTIONS}

We have attempted to present the many facets of how food recommendation technology has been proposed and used in prior work to influence healthy food choice. In addition, we have weaved in gaps and potential opportunities for future research throughout the narrative. In this section, we summarize how the contexts and approaches studied in this article can be utilized together and identify the gaps (see Table 4 for summary) and resulting potential opportunities for future work.

\subsection{Leveraging Multiple Food Choice Consideration}

Sections 4.1 and 4.2 present the intersection of the themes (categories) of different food choice technologies and their context of use in different scenarios for encouraging healthy food decisions, respectively. The themes correspond to modeling and analyzing user preferences (person-related factors) or the flavor and nutritional components of recommended food (food properties' factors) or the intersection of consumer, food, and environment (environmental factors). The context of use corresponds to how the these food recommendation systems can be used - in grocery shopping, social interactions, or for personal usage and tracking. Thus, any food recommendation technology research must combine the multiple variables stemming from these themes and contexts to more reliably recommend healthy and preferential recipes for the user.

These themes and context of use have great overlap. Thus, they may be combined as system attributes (factors) to produce improved nutrition-based systems. Person-related factors, such as sensory perception [25], would work well with the grocery shopping context, as knowing the flavor of a particular meal might indicate whether the user (a comsumer) would enjoy it. The consumer could then be engaged through technology-enabled communication about taste. Flavor has already been used in recommendation models, as it is important in the decisionmaking process for consuming meals [42]. Understanding the consumer's health cognition (i.e., aptitude to eat healthy) would also aid in grocery selection, as the application could narrow meals to meet the consumer's health needs and preferences similar to what was done by Wayman and Madhvanath using grocery receipts to identify health cognitions that could persuade grocery shoppers to eat healthier [75]. Furthermore, with respect to the social context theme, systems can be built to consider how social variables or cultural traits affect a consumer's 
Table 4. Gaps Found Throughout Each Section

\begin{tabular}{l|l}
\hline \multicolumn{1}{c}{ Review Section } & \multicolumn{1}{c}{ Gaps and Opportunities Identified } \\
\hline Themes & $\begin{array}{l}\text { Environmental constraints on consumers, such as distance to super- } \\
\text { markets, may have an impact on consumers' eating habits based on } \\
\text { geographic location and personal constraints, such as cultural charac- } \\
\text { teristics, may limit dietary consumption. Thus, both should be consid- } \\
\text { ered for food choice technologies. }\end{array}$ \\
\hline Context of use & $\begin{array}{l}\text { More focus is needed on the grocery shopping aspect as healthy food } \\
\text { availability and price are a major fact in making healthy food decisions. } \\
\text { From the social aspect, family and friends eating habits may also need } \\
\text { to be considered more in recommendations. }\end{array}$ \\
\hline Technological approaches & $\begin{array}{l}\text { Social variables, such as social support and influence, may have an ef- } \\
\text { fect on consumer eating habits. Thus, they could be potential features } \\
\text { for recommendations. }\end{array}$ \\
\hline Dataset types & $\begin{array}{l}\text { It would be helpful to understand how combining or interchangeably } \\
\text { utilizing datasets from various sources and those from food recipe web- } \\
\text { sites could be used to build new models and interfaces that can reflect } \\
\text { information from both datasets. }\end{array}$ \\
\hline Commonly used evaluation metrics & $\begin{array}{l}\text { More focus is needed on evaluation of recommendation systems utiliz- } \\
\text { ing users and getting their feedback on the system. }\end{array}$ \\
\hline
\end{tabular}

choices $[17,55,60]$. Cultural information can provide insight into a consumer's food habits, as different cultures may exhibit varying eating habits. For example, the Italian food combination of salty and dry ham with moist sweet melon was inspired by the rules of eating opposites further informed by temperature and moisture.

Environmental factors, such as food availability, should also be considered in system design, as understanding consumer's geographical context, such as living in food deserts, can impact food decisions [36]. If a consumer has financial limitations while also having cultural constraints on food consumption, a thorough recommendation system can recommend meals that fit their constraints and/or preferences. Examples of a recommendation model capable of considering such heterogeneous data are factorization models as well as deep learning-based recommendation models $[6,84]$.

As we imagine the confluence of these themes and contexts in the design of food recommendation systems, it is also important for researchers and practitioners to consider drawbacks associated with considering a vast amount of features (in data and in application) for predictions and user experience. In real-world applications, users would have to specify their preferences and constraints to the application, which then become the feature vectors to train the machine learning algorithms for generating predictions and recommendations on food choices. Thus, it is essential to consider what are the best ways to elicit user preference and constraints in the most efficient manner, as a person's attention span can be limited to 8 seconds before that person gets distracted [28]. For example, if users come to a meal platform application to gain access to healthy food recommendations, they would need to input a vast amount of information to ensure that the recommendation algorithm accommodates their preferences. If they have to input too much information, they may leave the application for something less time-consuming. While some variables may not be as important to the recommendation system (e.g., gender) others, such as Meal Rating, are particularly necessary as ratings can easily infer attraction towards specific meals. Therefore, it is important to consider trade-offs and which set of features is the most important to make recommendations, as utilizing all features may prove detrimental to the consumer experience. We can see, based 
on studies by Forwood et al. [25], that food knowledge is important as marketing tactics can cause consumers to choose an unhealthy snack over a healthy one even if they know that what they are consuming is unhealthy. There are many factors to consider when developing food technologies and specifically those that focus on health. It would be useful to consider techniques that can take in heterogeneous data from users and provide them with helpful information on healthy eating in return. We discuss a potential method for this as a future opporunity in the next subsection.

\subsection{Future Opportunities}

This survey, while providing a summary of prior work, also provides gaps and resulting opportunities for researchers to understand and gauge the importance of applying technologically based approaches to encouraging healthy food decisions. The various technological and evaluation approaches that have been discussed, particularly machine learning-based methods and user studies, may be key to providing users with healthy food choices and encouraging those decisions. Advances in machine learning algorithms that can integrate data stemming from various contexts and themes can lead to more informed predictions about healthy meals and habits, which might have a higher stickiness as they are incorporating the person/social/environmental factors as well as the context of use from grocery shopping to self-tracking. Improvements in machine learning through more advanced recommendation systems and methods such as deep learning provide researchers with tools to make healthy food predictions for consumers. In particular, a modeling and recommendation approach that accounts for the complexity of the problem, such as heterogeneous network models, would be useful. Heterogeneous networks [21] can account for a variety of data types and lead to informed decisions for the consumer on food choices. Given the human interaction and consumption of the output of the machine learning models, it is also important to ensure interpretability of the developed machine learning algorithms such that the end consumer can have a higher level of understanding about why certain recommendations are more relevant for that user.

It is also not sufficient to develop machine learning algorithms and evaluate them empirically for the predictability on recommendations. It is equally important to develop intuitive user interfaces that are engaging with the end-user and incorporate an evaluation framework that incorporates results from user studies in design and development. These user interfaces can combine different contexts, such as grocery shopping and personal tracking/monitoring, to ensure a deeper engagement with the user. As discussed, sensory perception (i.e., considering a user's taste) could be implemented on applications that show the unique tastes of groceries in the store. As was shown by [25], taste plays an important role in what users tend to purchase at grocery stores. Furthermore, the contexts combined with the consideration of themes such as person-related factors (e.g., sensory perception, health cognition, physical health, and social influence), properties of the food (e.g., nutrition composition), and environmental factors (e.g., food availability and eating environment) can be utilized in future health-related systems. Essentially, by taking variables related to the various contexts and considering variables related to each specific theme, the performance of food technology systems can be improved. Multiple variables, such as personal preferences, health cognition, food availability, food ratings, and demographic information, can be included in mobile applications simultaneously, such as social networks or food applications, classification algorithms, and recommendation algorithms. Veinot et al. also discussed social determinants of health, which included factors such as LGBT identity, occupation, health screenings, and public policies [72]. Understanding how consumer health is affected by social determinants can also benefit health-based recommendation systems as further feature information. However, these predictions can be made only with proper research into understanding which variables should be used as input to the model.

\section{CONCLUSION}

The goal of this review is to provide researchers with relevant and recent trends on how to best provide consumers with technological solutions for supporting healthy food choice. Our review examined the development 
of food choice technologies by identifying common themes, context of use, technologies, and research gaps found within the chosen papers. The themes were carefully organized into sections to describe studies that relate directly to the established themes and the context in which the themes are frequently used. The themes of the papers are portrayed as a grouping of the ideas/variables that the papers are intended to represent. We also discussed the various datasets found throughout the chosen papers for this review as well as evaluation metrics. It is crucial for researchers to define and understand the best metrics to use when evaluating their technologically based approaches as well as where to locate data in order to perform analysis. Through the analysis of the papers, we discovered that researchers solved some (but not all) of the problems related to healthy food choice by utilizing technological approaches such as machine learning, interface/app development, and social network analysis. By analyzing the evaluation results of all papers covered, we were able to give a clear path for researchers to perform future work. This path was enabled through our discussion of the limitations of current work and the gaps that failed to be addressed in prior studies. The authors hope that this review and its findings will follow with more research that can aid consumers in pursuing healthier food choices in order to prevent them from developing dietary and lifestyle-related illnesses, which include heart disease, obesity, high blood pressure, and diabetes.

\section{REFERENCES}

[1] Sofiane Abbar, Yelena Mejova, and Ingmar Weber. 2015. You tweet what you eat: Studying food consumption through Twitter. In CHI. ACM, 3197-3206.

[2] Ifeoma Adaji and Julita Vassileva. 2017. A gamified system for influencing healthy e-commerce shopping habits. In Adjunct Publication of the 25th Conference on User Modeling, Adaptation and Personalization. 398-401.

[3] Giuseppe Agapito, Mariadelina Simeoni, Barbara Calabrese, Pietro Hiram Guzzi, Giorgio Fuiano, and Mario Cannataro. 2017. DIETOS: A recommender system for health profiling and diet management in chronic diseases. In HealthRecSys@RecSys. 32-35.

[4] AHA. 2014. American Heart Association statistical report tracks global figures for first time. AHA. https://www.heart.org/en/news/ 2018/05/01/american-heart-association-statistical-report-tracks-global-figures-for-first-time.

[5] Junho Ahn, James Williamson, Mike Gartrell, Richard Han, Qin Lv, and Shivakant Mishra. 2015. Supporting healthy grocery shopping via mobile augmented reality. TOMCCAP 12 (2015), 16:1-16:24.

[6] Mehmet Aktukmak, Yasin Yilmaz, and Ismail Uysal. 2019. A probabilistic framework to incorporate mixed-data type features: Matrix factorization with multimodal side information. Neurocomputing 367 (2019), 164-175.

[7] Ahmed Al-Nazer and Tarek Helmy. 2012. Toward a cross-cultural and cross-language multi-agent recommendation model for food and nutrition. In IEEE/WIC/ACM International Conferences on Web Intelligence and Intelligent Agent Technology 3 (2012), 245-249.

[8] Eman Almonani, Wahidah Husain, Oh Ying San, Ammar ALmomani, and Mohammed Azmi Al-Betar. 2014. Mobile game approach to prevent childhood obesity using persuasive technology. In International Conference on Computer and Information Sciences (ICCOINS'14). $1-5$.

[9] Kerry Shih-Ping Chang, Catalina Danis, and Robert G. Farrell. 2014. Lunch line: Using public displays and mobile devices to encourage healthy eating in an organization. In Proceedings of the 2014 ACM International foint Conference on Pervasive and Ubiquitous Computing. 823-834.

[10] Beenish M. Chaudhry, Christopher L. Schaefbauer, Ben Jelen, Katie A. Siek, and Kay Connelly. 2016. Evaluation of a food portion size estimation interface for a varying literacy population. In Proceedings of the 2016 CHI Conference on Human Factors in Computing Systems. 5645-5657.

[11] Mei-Yun Chen, Yung-Hsiang Yang, Chia-Ju Ho, Shih-Han Wang, Shane-Ming Liu, Eugene T. Y. Chang, Che-Hua Yeh, and Ming Ouhyoung. 2012. Automatic Chinese food identification and quantity estimation. In SIGGRAPH Asia 2012 Technical Briefs. 1-4.

[12] Hao Cheng, Markus Rokicki, and Eelco Herder. 2017. The influence of city size on dietary choices. In Proceedings of the 25th Conference on User Modeling, Adaptation and Personalization (UMAP'17). ACM, New York, NY, 359-360. https://doi.org/10.1145/3079628.3079641

[13] Munmun De Choudhury, Sanket S. Sharma, and Emre Kiciman. 2016. Characterizing dietary choices, nutrition, and language in food deserts via social media. In Proceedings of the 19th Acm Conference on computer-supported cooperative work \& social computing. 11571170 .

[14] Chia-Fang Chung, Elena Agapie, Jessica Schroeder, Sonali R. Mishra, James Fogarty, and Sean A. Munson. 2017. When personal tracking becomes social: Examining the use of Instagram for healthy eating. In Proceedings of the 2017 CHI Conference on Human Factors in Computing Systems. 1674-1687.

[15] Rob Comber, Jack Weeden, Jennifer Hoare, Stephen Lindsay, Gemma Teal, Alastair Macdonald, Lisa Methven, Paula Moynihan, and Patrick Olivier. 2012. Supporting visual assessment of food and nutrient intake in a clinical care setting. In Proceedings of the SIGCHI Conference on Human Factors in Computing Systems (CHI'12). ACM, New York, NY, 919-922. https://doi.org/10.1145/2207676.2208534 
[16] Aron Culotta. 2014. Estimating county health statistics with Twitter. In Proceedings of the SIGCHI Conference on Human Factors in Computing Systems (CHI'14). ACM, New York, NY, 1335-1344. https://doi.org/10.1145/2556288.2557139

[17] Tiago Oliveira Cunha, Ingmar Weber, and Gisele Lobo Pappa. 2017. A warm welcome matters! The link between social feedback and weight loss in /r/loseit. In Proceedings of the 26th International Conference on World Wide Web Companion. 1063-1072.

[18] Michael J. Devlin, Susan Z. Yanovski, and G. Terence Wilson. 2000. Obesity: What mental health professionals need to know. American Journal of Psychiatry 157, 6 (2000), 854-866.

[19] Kristen Nicole DiFilippo, Wen-Hao Huang, Juan E. Andrade, and Karen M. Chapman-Novakofski. 2015. The use of mobile apps to improve nutrition outcomes: A systematic literature review. Journal of Telemedicine and Telecare 215 (2015), 243-253.

[20] Daniel Doerr, Kun Hu, Sondra R. Renly, Stefan Edlund, Matthew A. Davis, James H. Kaufman, Justin Lessler, Matthias Filter, Annemarie Käsbohrer, and Bernd Appel. 2012. Accelerating investigation of food-borne disease outbreaks using pro-active geospatial modeling of food supply chains. In Proceedings of the First ACM SIGSPATIAL International Workshop on Use of GIS in Public Health. 44-47.

[21] Yuxiao Dong, Nitesh V. Chawla, and Ananthram Swami. 2017. metapath2vec: Scalable representation learning for heterogeneous networks. In Proceedings of the 23rd ACM SIGKDD International Conference on Knowledge Discovery and Data Mining. 135-144.

[22] David Elsweiler and Morgan Harvey. 2015. Towards automatic meal plan recommendations for balanced nutrition. In Proceedings of the 9th ACM Conference on Recommender Systems. 313-316.

[23] David Elsweiler, Christoph Trattner, and Morgan Harvey. 2017. Exploiting food choice biases for healthier recipe recommendation. In Proceedings of ACM SIGIR Conference on Research and Development in Information Retrieval. ACM, 575-584

[24] Hannah Forster, Marianne C. Walsh, Clare B. O’Donovan, Clara Woolhead, Caroline McGirr, Edward Daly, Richard O’Riordan, Carlos Celis-Morales, Rosalind Fallaize, Anna L. Macready, Cyril F. M. Marsaux, Santiago Navas-Carretero, Rodrigo San-Cristobal, Silvia Kolossa, Kai Hartwig, Christina Mavrogianni, Lydia Tsirigoti, Christina P. Lambrinou, Magdalena Godlewska, Agnieszka Surwilĺlo, Ingrid M. F. Gjelstad, Christian A. Drevon, Yannis Manios, Iwona Traczyk, J. Alfredo Martínez, Wim H. M. Saris, Hannelore Daniel, Julie A. Lovegrove, John C. Mathers, Michael J. Gibney, Eileen R. Gibney, and Lorraine Brennan. 2016. A dietary feedback system for the delivery of consistent personalized dietary advice in the web-based multicenter Food4Me study. fournal of Medical Internet Research 18, 6 (2016), e5620.

[25] Suzanna E. Forwood, Alexander D. Walker, Gareth J. Hollands, and Theresa M. Marteau. 2013. Choosing between an apple and a chocolate bar: The impact of health and taste labels. PLoS One 8, 10 (2013), e77500.

[26] International Food Information Council Foundation. 2017. 2017 Food and Health Survey. International Food Information Council Foundation. https://foodinsight.org/2017-food-and-health-survey-a-healthy-perspective-understanding-american-food-values.

[27] Jill Freyne and Shlomo Berkovsky. 2010. Recommending food: Reasoning on recipes and ingredients. In International Conference on User Modeling, Adaptation, and Personalization. Springer, 381-386.

[28] Chris Galloway. 2017. Blink and they're gone: PR and the battle for attention. Public Relations Review 43, 5 (2017), 969-977.

[29] Mouzhi Ge, Mehdi Elahi, Ignacio Fernández-Tobías, Francesco Ricci, and David Massimo. 2015. Using tags and latent factors in a food recommender system. In Proceedings of the 5th International Conference on Digital Health 2015. 105-112.

[30] Mouzhi Ge, Francesco Ricci, and David Massimo. 2015. Health-aware food recommender system. In Proceedings of the 9th ACM Conference on Recommender Systems. 333-334.

[31] Penny Gordon-Larsen. 2014. Food availability/convenience and obesity. Advances in Nutrition 5, 6 (2014), 809-817. https://doi.org/10 3945/an.114.007070 arXiv:/oup/backfile/content_public/journal/advances/5/6/10.3945_an.114.007070/6/809.pdf.

[32] Ross Gore, Saikou Y. Diallo, Jose Padilla, and David Meyre. 2015. You are what you tweet: Connecting the geographic variation in America's obesity rate to Twitter content. PloS one 10, 9 (2015), e0133505.

[33] Andrea Grimes, Vasudhara Kantroo, and Rebecca E. Grinter. 2010. Let’s play!: Mobile health games for adults. In UbiComp.

[34] Asela Gunawardana and Guy Shani. 2009. A survey of accuracy evaluation metrics of recommendation tasks. Fournal of Machine Learning Research 10 (2009), 2935-2962.

[35] Anna Herforth and S. Shaikh Saubiya Ahmed. 2015. The food environment, its effects on dietary consumption, and potential for measurement within agriculture-nutrition interventions. Food Security 7 (2015), 505-520.

[36] Julia M. Hormes and Martha A. Niemiec. 2017. Does culture create craving? Evidence from the case of menstrual chocolate craving. In PLoS One.

[37] Patrick D. Howell, Layla D. Martin, Hesamoddin Salehian, Chul Lee, Kyler M. Eastman, and Joohyun Kim. 2016. Analyzing taste preferences from crowdsourced food entries. In Proceedings of the 6th International Conference on Digital Health Conference. 131-140.

[38] Jinhyun Jun, Susan W. Arendt, and Juhee Kang. 2016. Understanding customers' healthful food selection at restaurants: Roles of attitude, gender, and past experience. fournal of Foodservice Business Research 19, 2 (2016), 197-212.

[39] Vaiva Kalnikaité, Jon Bird, and Yvonne Rogers. 2012. Decision-making in the aisles: Informing, overwhelming or nudging supermarket shoppers? Personal and Ubiquitous Computing 17 (2012), 1247-1259.

[40] Egon P. Köster. 2009. Diversity in the determinants of food choice: A psychological perspective. Food Quality and Preference 20, 2 (2009), $70-82$.

[41] Yu-Wen Lo, Qiangfu Zhao, Yu-Hsien Ting, and Rung-Ching Chen. 2015. Automatic generation and recommendation of recipes based on outlier analysis. In IEEE 7th International Conference on Awareness Science and Technology (iCAST'15). IEEE, 216-221. 
[42] Xuehui Mao, Shizhong Yuan, Weimin Xu, and Darning Wei. 2016. Recipe recommendation considering the flavor of regional cuisines. In International Conference on Progress in Informatics and Computing (PIC'16). 32-36.

[43] Yelena Mejova, Hamed Haddadi, Anastasios Noulas, and Ingmar Weber. 2015. FoodPorn: Obesity patterns in culinary interactions. In Proceedings of the 5th International Conference on Digital Health 2015. 51-58.

[44] Weiqing Min, Bing-Kun Bao, Shuhuan Mei, Yaohui Zhu, Yong Rui, and Shuqiang Jiang. 2017. You are what you eat: Exploring rich recipe information for cross-region food analysis. IEEE Transactions on Multimedia 20, 4 (2017), 950-964.

[45] Jon Noronha, Eric Hysen, Haoqi Zhang, and Krzysztof Z. Gajos. 2011. Platemate: Crowdsourcing nutritional analysis from food photographs. In Proceedings of the 24th Annual ACM Symposium on User Interface Software and Technology (UIST'11). ACM, New York, NY, 1-12. https://doi.org/10.1145/2047196.2047198

[46] Monica Marina Nour, Juliana Chen, and Margaret A. Allman-Farinelli. 2016. Efficacy and external validity of electronic and mobile phone-based interventions promoting vegetable intake in young adults: Systematic review and meta-analysis. Fournal of Medical Internet Research 18, 4 (2016), e5082.

[47] Dimitrios Ntalaperas, Efthimios Bothos, Konstantinos Perakis, Babis Magoutas, and Gregoris Mentzas. 2015. DISYS: An intelligent system for personalized nutritional recommendations in restaurants. In Proceedings of the 19th Panhellenic Conference on Informatics. 382-387.

[48] Joongsin Park, Bon-Chang Koo, Jun-Dong Cho, and Byung-Chull Bae. 2015. SnackBreaker: A game promoting healthy choice of snack foods. In Proceedings of the 2015 Annual Symposium on Computer-Human Interaction in Play. 673-678.

[49] Nora Ptakauskaite, Priscilla Chueng-Nainby, and Helen Pain. 2016. Supporting social innovation in children: Developing a game to promote health eating. In Proceedings of the The 15th International Conference on Interaction Design and Children. 688-693.

[50] Wolfgang H. Reitberger, Wolfgang Spreicer, and Geraldine Fitzpatrick. 2014a. Nutriflect: Reflecting collective shopping behavior and nutrition. In Proceedings of the 32nd Annual ACM Conference on Human Factors in Computing Systems (CHI'14). ACM, New York, NY, 3309-3318. https://doi.org/10.1145/2556288.2557384

[51] Wolfgang H. Reitberger, Wolfgang Spreicer, and Geraldine Fitzpatrick. 2014b. Situated and mobile displays for reflection on shopping and nutritional choices. Personal and Ubiquitous Computing 18 (2014b), 1721-1735.

[52] Jaclyn Rich, Hamed Haddadi, and Timothy M. Hospedales. 2016. Towards bottom-up analysis of social food. In Proceedings of the 6th International Conference on Digital Health Conference. 111-120.

[53] Christina A. Roberto, Boyd A. Swinburn, Corinna Hawkes, Terry T. Huang, Sérgio Antonio Araújo Costa, Marice Ashe, Lindsey Zwicker, John Cawley, and Kelly D. Brownell. 2015. Patchy progress on obesity prevention: Emerging examples, entrenched barriers, and new thinking. Lancet 3859985 (2015), 2400-2409.

[54] Markus Rokicki, Eelco Herder, and Christoph Trattner. 2017. How editorial, temporal and social biases affect online food popularity and appreciation. In ICWSM. 192-200.

[55] Sina Sajadmanesh, Sina Jafarzadeh, Seyed Ali Ossia, Hamid R. Rabiee, Hamed Haddadi, Yelena Mejova, Mirco Musolesi, Emiliano De Cristofaro, and Gianluca Stringhini. 2017. Kissing cuisines: Exploring worldwide culinary habits on the web. In Proceedings of the 26th International Conference on World Wide Web Companion. 1013-1021.

[56] Kate M. Scott, Ronny Bruffaerts, Greg E. Simon, Jordi Alonso, Matthias Angermeyer, Giovanni De Girolamo, Koen Demyttenaere, Isabelle Gasquet, Josep Maria Haro, Elie Karam, et al. 2008. Obesity and mental disorders in the general population: Results from the World Mental Health Surveys. International fournal of Obesity 32, 1 (2008), 192-200.

[57] Sanket S. Sharma and Munmun De Choudhury. 2015. Measuring and characterizing nutritional information of food and ingestion content in Instagram. In Proceedings of the 24th International Conference on World Wide Web. 115-116.

[58] Sangeetha Shekar, Prashant Nair, and Abdelsalam Helal. 2003. iGrocer - A ubiquitous and pervasive smart grocery shopping system. In Proceedings of the 2003 ACM symposium on Applied Computing. 645-652.

[59] Chokdee Siawsolit, Sarun Seepun, Jennifer Choi, An Do, and Yu Kao. 2017. Personalized assistant for health-conscious grocery shoppers. In International Conference on Persuasive Technology. Springer, 95-106.

[60] Thiago H. Silva, Pedro O. S. Vaz de Melo, Jussara M. Almeida, Mirco Musolesi, and Antonio Alfredo Ferreira Loureiro. 2014. You are what you eat (and drink): Identifying cultural boundaries by analyzing food and drink habits in foursquare. In Eighth International AAAI Conference on Weblogs and Social Media.

[61] Jan-Benedict E. M. Steenkamp. 1993. Food consumption behavior. E - European Advances in Consumer Research 1 (1993), 401-409.

[62] F. Marijn Stok, Stefan Hoffmann, Dorothee Volkert, Heiner Boeing, Regina Ensenauer, Marta Stelmach-Mardas, Eva Kiesswetter, Alisa Weber, Harald Rohm, Nanna Lien, et al. 2017. The DONE framework: Creation, evaluation, and updating of an interdisciplinary, dynamic framework 2.0 of determinants of nutrition and eating. PLoS One 12, 2 (2017), e0171077.

[63] Claudia Symmank, Robert Mai, Stefan Hoffmann, F. Marijn Stok, Britta Renner, Nanna Lien, and Harald Rohm. 2017. Predictors of food decision making: A systematic interdisciplinary mapping (SIM) review. Appetite 110 (2017), 25-35.

[64] Toshiki Takeuchi, Tatsuya Fujii, Takuji Narumi, Tomohiro Tanikawa, and Michitaka Hirose. 2015. Considering individual taste in social feedback to improve eating habits. In IEEE International Conference on Multimedia \& Expo Workshops (ICMEW'15). IEEE, 1-6.

[65] ChunYuen Teng, Yu-Ru Lin, and Lada A. Adamic. 2012. Recipe recommendation using ingredient networks. In Proceedings of the 4th Annual ACM Web Science Conference. 298-307. 
[66] Emma Tonkin, Julie Brimblecombe, and Thomas Philip Wycherley. 2017. Characteristics of smartphone applications for nutrition improvement in community settings: A scoping review. Advances in Nutrition 8, 2 (2017), 308-322.

[67] Thi Ngoc Trang Tran, Müslüm Atas, Alexander Felfernig, and Martin Stettinger. 2017. An overview of recommender systems in the healthy food domain. Journal of Intelligent Information Systems (2017), 1-26.

[68] Christoph Trattner and David Elsweiler. 2017. Food recommender systems: Important contributions, challenges and future research directions. CoRR abs/1711.02760

[69] Christoph Trattner and David Elsweiler. 2017. Investigating the healthiness of Internet-sourced recipes: Implications for meal planning and recommender systems. In Proceedings of the 26th International Conference on World Wide Web. 489-498.

[70] Christoph Trattner, Markus Rokicki, and Eelco Herder. 2017. On the relations between cooking interests, hobbies and nutritional values of online recipes: Implications for health-aware recipe recommender systems. In Adjunct Publication of the 25th Conference on User Modeling, Adaptation and Personalization. 59-64.

[71] Tsuguya Ueta, Masashi Iwakami, and Takayuki Ito. 2011. A recipe recommendation system based on automatic nutrition information extraction. In International Conference on Knowledge Science, Engineering and Management. Springer, 79-90.

[72] Tiffany C. Veinot, Jessica S. Ancker, Heather Cole-Lewis, Elizabeth D. Mynatt, Andrea G. Parker, Katie A. Siek, and Lena Mamykina 2019. Leveling up: On the potential of upstream health informatics interventions to enhance health equity. Medical Care 57 (2019), S108-S114.

[73] Claudia Wagner, Philipp Singer, and Markus Strohmaier. 2014. Spatial and temporal patterns of online food preferences. In WWW.

[74] Dong Wang, Lance Kaplan, and Tarek Abdelzaher. 2014. Maximum likelihood analysis of conflicting observations in social sensing. ACM Transactions on Sensor Networks 10, 2, Article 30.

[75] Elizabeth Wayman and Sriganesh Madhvanath. 2015. Nudging grocery shoppers to make healthier choices. In Proceedings of the 9th ACM Conference on Recommender Systems. 289-292.

[76] Robert West, Ryen W. White, and Eric Horvitz. 2013. From cookies to cooks: Insights on dietary patterns via analysis of web usage logs. In Proceedings of the 22nd international conference on World Wide Web. 1399-1410.

[77] World Health Organization. 2017. Blood Pressure. WHO. https://newsroom.heart.org/news/.

[78] World Health Organization. 2017. Global and regional food consumption patterns and trends. WHO. https://www.who.int/ dietphysicalactivity/publications/trs916/en/gsfao_global.pdf.

[79] World Health Organization. 2017. Obesity and overweight. WHO. https://www.who.int/news-room/fact-sheets/detail/obesity-andoverweight.

[80] Yi-Jing Wu and Wei-Guang Teng. 2011. An enhanced recommendation scheme for online grocery shopping. In IEEE 15th International Symposium on Consumer Electronics (ISCE'11). 410-415.

[81] Longqi Yang, Cheng-Kang Hsieh, Hongjian Yang, John P. Pollak, Nicola Dell, Serge J. Belongie, Curtis Cole, and Deborah Estrin. 2017. Yum-Me: A personalized nutrient-based meal recommender system. ACM Transactions on Information Systems 36 (2017), 7:1-7:31.

[82] Shu-Ching Yang, Yi-Fang Luo, and Chia-Hsun Chiang. 2017. The associations among individual factors, eHealth literacy, and healthpromoting lifestyles among college students. Journal of Medical Internet Research. 19, 1 (2017), e15.

[83] Xinyue Ye and Jay Lee. 2016. Integrating geographic activity space and social network space to promote healthy lifestyles. SIGSPATIAL Special 8 (2016), 20-33.

[84] Baolin Yi, Xiaoxuan Shen, Hai Liu, Zhaoli Zhang, Wei Zhang, Sannyuya Liu, and Naixue Xiong. 2019. Deep matrix factorization with implicit feedback embedding for recommendation system. IEEE Transactions on Industrial Informatics 15, 8 (2019), 4591-4601.

[85] Fuzheng Zhang, Kai Zheng, Nicholas Jing Yuan, Xing Xie, Enhong Chen, and Xiaofang Zhou. 2015. A novelty-seeking based dining recommender system. In Proceedings of the 24th International Conference on World Wide Web. 1362-1372.

Received February 2019; revised January 2021; accepted October 2021 